\title{
ACUTE GENERALIZED EXANTHEMATOUS PUSTULOSIS INDUCED BY OLANZAPINE
}

\author{
Susanne Christen', Félix Gueissaz ${ }^{2}$, Romain Anex ${ }^{3}$, Daniele Fabio Zullino \\ Psychiatric Clinic Préfargier, Marin, Switzerland ${ }^{1}$; Private dermatologic practice, Neuchâtel, Switzerland ${ }^{2}$; Laboratory \\ Meditest, Morges, Switzerland ${ }^{3}$; University Hospitals of Geneva, Switzerland: Division of Substance Abuse ${ }^{4}$ \\ Summary: The case of a 56 years old man is presented, who developed acute generalized exanthematous pustulosis 5 days \\ after the introduction of olanzapine $10 \mathrm{mg}$. Multiple 1-mm pustules appeared on the whole body, concentrated especially on \\ her neck and face. Within 2 days, the eruption was increasingly accompanied by erythema and pruritus. No fever, chills, nau- \\ sea, vomiting, arthralgias or myalgias were recorded. The diagnosis was corroborated by hystopathology. After 7 days of treat- \\ ment, olanzapine and valproate were stopped. Concomitantly, cetirizine $20 \mathrm{mg}$ p.o. and methylprednisolone $500 \mathrm{mg}$ i.v. were \\ given once. During the following week betamethasone cream was applied, and the pustular eruption resolved completely.
}

Key words: Antipsychotic Agents; Exanthema; Adverse effects; Olanzapine

\section{Introduction}

Acute generalized exanthematous pustulosis (AGEP) is caused in $90 \%$ of the cases by systemic medication. It typically presents with at least dozens of non-follicular sterile pustules occurring on a diffuse, edematous erythema predominantly in the folds and/or on the face. Patients may report a burning or itching sensation. The onset is most often acute and pustules typically resolve spontaneously within less than 15 days. It typically takes 1-3 weeks after introduction of a new drug until skin symptoms arise, which has been suggested to be the result of a primary sensitization. A second group of patients, especially those under antibacterial drugs, may present skin symptoms after a shorter latency of 2-3 days. The overall prognosis is good although high fever or superinfection may sometimes lead to life-threatening situations in patients of old age or poor general condition. Histopathology characteristically reveals spongiform subcorneal and/or intraepidermal pustules, a marked edema of the papillary dermis, and eventually vasculitis, eosinophils and/or focal necrosis of keratinocytes.

A wide range of drugs has been reported of causing the reaction, especially antibiotics, calcium channel blockers, carbamazepine and paracetamol. One case of AGEP induced by the newer antipsychotic olanzapine has recently been published. We report a second case of olanzapine induced pustular eruption documented within the drug safety program in psychiatry AMSP (Arzneimittelsicherheit in der Psychiatrie).

\section{Case report}

Mrs A, 56 years old, Caucasian, has a psychiatric history since the age of 25 (dysthymic and subsequently depressive), and a bipolar disorder has been diagnosed at the age of 52. She had no history of severe somatic disorder, and in particular no record of allergic reactions. Being treated since 7 month with fluoxetine $20 \mathrm{mg}$ and mirtazapine 30 $\mathrm{mg}$, she was currently hospitalized due to a mixed episode, presenting increased drive, logorrhea, disturbances of concentration, and shortened sleep, with concomitant periods of dysphoria and lack of confidence.

At first, a treatment with midazolam $7.5 \mathrm{mg}$, clorazepate $5 \mathrm{mg}$ and clotiapine $40 \mathrm{mg}$ was added. During the first 7 days of hospitalization, the symptomatology worsened, the patient presenting increasingly flight of ideas, exaggerated self-esteem, and especially irritability. Olanzapine 10 $\mathrm{mg}$ and valproate $1000 \mathrm{mg}$ were added to the preexisting medication, and clotiapine discontinued.

Five days after the introduction of olanzapine and valproate, multiple 1-mm pustules appeared on the whole body, concentrated especially on her neck and face (Fig. 1). Within 2 days, the eruption was increasingly accompanied by erythema and pruritus. No fever, chills, nausea, vomiting, arthralgias or myalgias were recorded.

A biopsy was performed during a dermatological consilium. Histopathology revealed a slight acanthosis, exocytosis of lymphocytes, and intraepidermic polynuclear granulocytes. Locally, the polynuclear granulocytes formed subcorneal pustules. Furthermore, an inflammatory in- 
filtration with polynuclear granulocytes and lymphocytes surrounding the follicular structures, as well as a papillary dermal edema were found.

After 7 days of treatment, olanzapine and valproate were stopped. Concomitantly, cetirizine $20 \mathrm{mg}$ p.o. and methylprednisolone $500 \mathrm{mg}$ i.v. were given once. During the following week betamethasone cream was applied, and the pustular eruption resolved completely.

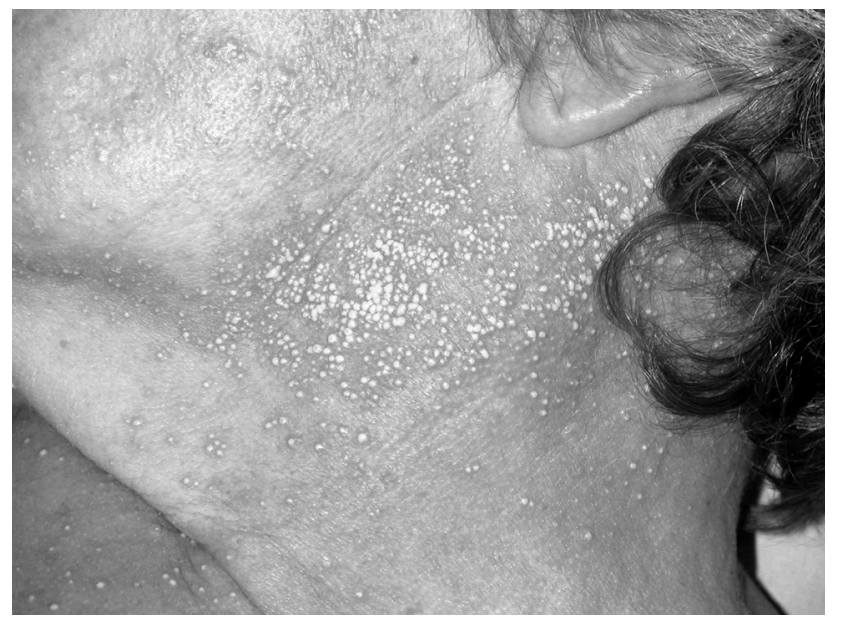

Fig. 1: Left neck with multiple 1 to $2 \mathrm{~mm}$ pustules on erythematous and edematous plaques.

\section{Discussion}

The exact mechanism of AGEP caused by drugs remains uncertain. It has, among others, bee proposed that the drug or its metabolites interact with antibodies leading to immune complexes, which in turn may activate the com- plement system, leading to the chemoattraction of neutrophils.

AGEP has been associated with various medications. We report the second case induced by olanzapine. The histology was similar to that in previous reports of pustular drug eruption, and especially to the previously published case with olanzapine. A major role of olanzapine in causing the eruption is strongly supported by the onset, which was temporally strongly associated with the start of olanzapine, and the remission that followed discontinuance of the drug. The same may be true for valproate, which was introduced and discontinued simultaneously to olanzapine. Whereas no previous AGEP has been reported for valproate, a contributing role therefore seeming less likely, it cannot, conversely, definitely been ruled out.

\section{References}

1. Adams BB, Mutasim DF. Pustular eruption induced by olanzapine, a novel antipsychotic agent. J Am Acad. Dermatol. 1999:41(5 Pt 2): 851-853.

2. Sidoroff A, Halevy S, Bavinck JN, Vaillant L, Roujeau JC. Acute generalized exanthematous pustulosis (AGEP - a clinical reaction pattern. J Cutan. Pathol. 2001;28(3):113-119.

3. Wakelin SH, James MP. Diltiazem-induced acute generalised exanthematous pustulosis. Clin. Exp Dermatol. 1995;20(4)341-344.

Submitted December 2005.

Accepted February 2006.

Dr. Daniele Zullino, Service d'abus de substances, Hôpitaux Universitaires de Genève, Rue verte 2,

Genève, Switzerland. e-mail: daniele.zullino@hcuge.ch 